\title{
Towards Ergonomic Control of Human-Robot Co-Manipulation and Handover
}

\author{
Luka Peternel $^{1 *}$, Wansoo Kim ${ }^{1 *}$, Jan Babič ${ }^{2}$ and Arash Ajoudani ${ }^{1}$
}

\begin{abstract}
In this paper, we propose a novel method for the control of human-robot co-manipulation that takes into account the ergonomic requirements for the human co-worker. The robot uses a whole-body model of the human to optimise for the position of the co-manipulation task in the workspace. In this configuration, the overloading joint torques, i.e. the effects of an external load in human body joints, are minimised. In addition, the optimisation process includes several constraints, such as human arm manipulability properties, to ensure that the human has a good manipulation capacity in the given configuration. The main advantage of this approach is that the robot can potentially help to reduce the work-related strain and increase the productivity of the human co-worker. We validated the proposed method with experiments in two co-manipulation tasks: human using a device to polish an object that is delivered by the robot and a human-robot object handover.
\end{abstract}

\section{INTRODUCTION}

Driven by the goal of adding a certain level of flexibility in manufacturing systems, human-robot collaboration (HRC) has become one of the central research directions in past years [1]-[3]. In such scenarios, humans and robots come together and interact as pairs, which usually requires that the execution speed is limited [4]. Therefore, an important factor to demonstrate the economical sustainability of the robotic solutions in cage-free environments is to minimize the risks that cause further performance degradation. Health-related issues and the injuries caused in the workplaces are among the most critical to be considered, and a unified framework to guarantee workers well-being is highly needed.

The safety from the human point of view is often addressed by avoiding potential collisions [5], [6] and excessive impact forces with sophisticated control strategies [7], [8], or adhoc mechanisms [9]. Other studies proposed to use human demonstrations in an attempt to achieve a seamless collaborative behaviour of the robot [10]-[13]. However, as it is well known [14], [15], a common cause of injury during manipulating or carrying heavy objects, or performing repetitive tasks (e.g. drilling, polishing, etc.) is the mechanical overloading of body joints such as ankle, knee, and sacroiliac. Although such overloadings in workplaces are unavoidable, they are smaller in some configurations compared to the others [16], [17]. This work explains a

\footnotetext{
${ }^{1} \mathrm{HRI}^{2}$ Lab, Department of Advanced Robotics, Istituto Italiano di Tecnologia, Genoa, Italy, Email: luka.peternel@iit.it,wan-soo.kim@iit.it

${ }^{2}$ Department of Automation, Bio-cybernetics and Robotics, Jožef Stefan Institute, Ljubljana, Slovenia

${ }^{*}$ Contributed equally to the work.

This work was supported in part by the EU H2020 projects CogIMon (644727) and An.Dy (731540).
}

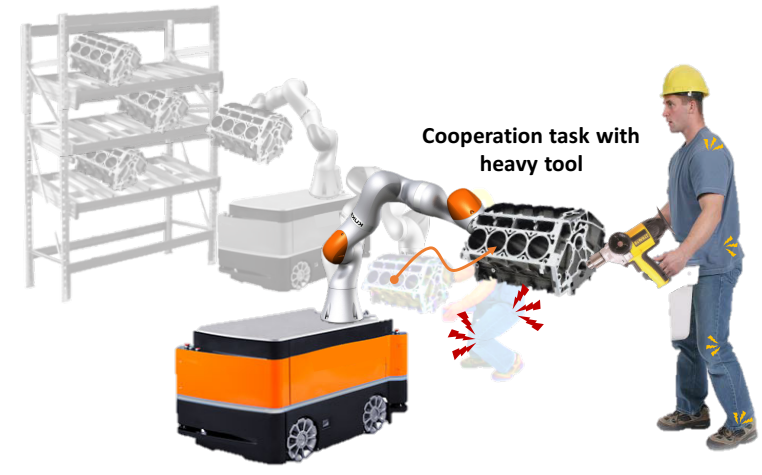

Fig. 1: Concept illustration: repetitive co-manipulation tasks can be performed in suitable human body poses that minimise the effect of overloading joint torque and maximise the human manipulation capacity.

potential way to keep the human in those configurations (see the illustration of the concept in Fig. 1).

To alleviate potential injury or chronic pathology risks due to the excessive mechanical overloadings of the joints, collaborating robots (CoBots) must be equipped with dynamic human state monitoring systems and anticipatory behaviours to guide the human counterparts towards more comfortable and ergonomic body poses. In this direction, we recently proposed a HRC framework that used a human body model and various sensory measurements to account for the overloading joint torques [18] in static poses of the human. The algorithm was based on the variations of the human Centre of Pressure (CoP), calculated from the difference between an off-line calibrated model and the measured one using an external force plate. The limitations of this approach is the static body-pose assumption and the need for an external force-plate.

To address the above-mentioned issues, in this paper we propose a novel dynamic human-robot collaboration control method to encourage and guide the human co-worker to perform the tasks in the most ergonomic way possible. To do so, a dynamic model of the human whole-body $\mathrm{CoP}$ is introduced. The model can automatically integrate the effect of an external mass (or interaction forces) from the robot endeffector measurements to estimate for the variations of the dynamic CoP in real-time. This consideration eliminates the need for an external force plate and makes the approach more practical. Finally, since the object co-manipulation tasks are considered in this study, the manipulation capacity of the human is taken into account in the optimisation procedure.

To validate the proposed method we performed experiments on a setup that included a KUKA Lightweight 
Robot arm and Pisa/IIT Softhand. We considered one comanipulation task, where the human had to used a hand-held machine to polish an object delivered by the robot, and one handover task 1 , where the robot had to handover an object to the human in a convenient location.

\section{METHOD}

A diagram of the proposed approach is shown in the right side of Fig. 2. The core of our approach is an on-line optimisation process that finds a human body configuration where the overloading joint torque ${ }^{2}$ are minimised. To do so, we perform a joint torque estimation that is based on on-line measurements of the human body configuration and a whole-body model of the human co-worker (see left side of Fig. 2), where parameters of the model are pre-estimated off-line.

The human arm manipulability is used as a constraint in this optimisation to ensure that the human arm has a good manipulation capacity in an optimised working position. In addition, other constraints related to a workspace limits of human and robot and the centre of pressure $(\mathrm{CoP})$ restriction are used to ensure safety and stability of the collaboration.

\section{A. Optimisation}

The proposed optimisation process can be formalised as

$$
\begin{gathered}
\min _{\mathbf{q}_{h}}\left\|\Delta \boldsymbol{\tau}^{T} \mathbb{W} \Delta \boldsymbol{\tau}\right\|, \\
\text { subject to }\left\{\begin{array}{c}
\mathbf{q}_{h} \in\left[\begin{array}{c}
\mathbf{q}_{L}, \quad \mathbf{q}_{U}
\end{array}\right] \\
\mathbf{C}_{P} \in \boldsymbol{\varepsilon}_{s} \\
\mathbf{p}_{\text {obj }}\left(\mathbf{q}_{h}\right) \in \mathbf{p}_{\text {task }} \\
w_{\text {min }} \leq w=\left|\operatorname{det}\left(\mathbf{J}_{a}\left(\mathbf{q}_{a}\right)\right)\right|,
\end{array}\right.
\end{gathered}
$$

where $\mathbf{q}_{h}$ is the human joint angle vector, $\Delta \boldsymbol{\tau}$ is a vector of overloading human joint torques and $\mathbb{W}$ can be used to weight the importance of joints. To calculate the overloading human joint torques $\Delta \tau$ we used a human whole-body model based on a modified statically equivalent serial chain (SESC) [18]. The details are explained in subsections II-B and IIC We used the ALGLIB optimisation library to solve the optimisation problem in (1).

The optimisation process (1) includes several constraints. The human joint angles $\mathbf{q}_{h}$ are constrained by upper and lower bounds $\mathbf{q}_{L}$ and $\mathbf{q}_{U}$. The CoP $\mathbf{C}_{P} \in \mathbb{R}^{2}$ of human is contained within the stable region $\boldsymbol{\varepsilon}_{S}$ (i.e. within the support polygon of feet). The position of the object $\mathbf{p}_{o b j}\left(\mathbf{q}_{h}\right)$ held by the robot is constrained within the feasible workspace of both the human and the robot $\mathbf{p}_{\text {task }}$.

Humans tend to exploit the configuration of their body and limbs to maximise kinematic and dynamic properties for the given task execution. Manipulability measures provide the information about the velocity and force production capacity of the limb endpoint in different configurations [19]. Directions of the best velocity and force axes can be obtained

\footnotetext{
${ }^{1}$ Handover can be considered as a special case of co-manipulation task.

${ }^{2}$ The overloading joint torque refers to the torque induced into the human joint by an external load.
}

from eigenvectors and eigenvalues of arm Jacobian $\mathbf{J}_{a}$. For example, if we need to produce or sustain high forces in a certain direction, the highest force manipulability vector can be aligned with that direction. In the opposite case, if we need to move the manipulated object fast in a certain direction, the highest velocity manipulability vector can be aligned with that direction. If a task requires a more complex manipulation that involves movements of the endpoint in many different directions, ideally the task should be performed around the configuration where the manipulability ellipsoid is isotropic. Hence, in our work, the position of the object that is co-manipulated is constrained by the human arm endpoint manipulability measure $w=\left|\operatorname{det}\left(\mathbf{J}_{a}\left(\mathbf{q}_{a}\right)\right)\right|$ that is defined by the human arm Jacobian $\mathbf{J}_{a} \in \mathbb{R}^{m \times l}$, where $m$ is the size of Cartesian space and $l$ is number of considered joints in the arm $\mathbf{q}_{a}$. The manipulability constraint is enforced by the threshold $w_{\text {min }}$. If $w_{\text {min }}=1$, the method will search for the optimal minimum overloading joint torques within human arm endpoint positions, where the manipulability ellipsoid is isotropic. In other cases, it will search within some region around isotropy.

\section{B. Human Whole-Body Model}

The human and the robot models are shown in the left side of Fig. 2. The human floating base frame $\Sigma_{0}$ was positioned at the pelvis link and connected from the inertial frame $\Sigma_{W}$ by a 6 virtual degrees of freedom (DoF) chain. We assumed the links are rigid and are articulated through $n$ revolute joints and defined a local reference frame $\Sigma_{i}$ at each joint. The robot base frame $\Sigma_{B}$ was defined at the base link of the robot. The endpoint of the human was at the human hand frame $\Sigma_{H}$ and the endpoint of the robot is at the robot tool frame $\Sigma_{T}$, both of which are connected to the external objects; e.g., tool, work pieces, etc.

The overloading joint torque can be defined in relation between the ground reaction forces (GRF) with and without the effect of an interaction force, and the CoP displacement due to the external load. The relation between $n_{k}$ contact points and the resultant contact force can be expressed by the Lagrangian based equation of motion with respect to $\Sigma_{W}$

$$
\mathbf{M}(\mathbf{q}) \ddot{\mathbf{q}}+\mathbf{C}(\mathbf{q}, \dot{\mathbf{q}}) \dot{\mathbf{q}}+\mathbf{G}(\mathbf{q})=\mathbf{S}^{T} \boldsymbol{\tau}+\sum_{i=1}^{n_{k}} \mathbf{J}_{p_{i}}(\mathbf{q})^{T} \mathbf{f}_{i},
$$

where $\mathbf{M}, \mathbf{C}$, and $\mathbf{G}$ represent the inertia matrix, the vector of centrifugal and Coriolis forces, and the vector of the gravity force, respectively. The generalised coordinates of the system are defined as $\mathbf{q}=\left[\begin{array}{lll}\mathbf{x}_{0}^{T} & \boldsymbol{\theta}_{0}^{T} & \mathbf{q}_{h}^{T}\end{array}\right]^{T} \in \mathbb{R}^{6+n}$, where $n$ is the number of DoF in the model. $\mathbf{x}_{0} \in \mathbb{R}^{3}$ and $\boldsymbol{\theta}_{0} \in \mathbb{R}^{3}$ represents the position and orientation of $\Sigma_{0}$ with respect to $\Sigma_{W}$, while $\mathbf{q}_{h} \in \mathbb{R}^{n}$ are angular positions of human joints. In addition, $\mathbf{S}=\left[\begin{array}{ll}\mathbf{0}_{n \times 6} & \mathbf{I}_{n \times n}\end{array}\right] \in \mathbb{R}^{n \times(n+6)}$ is the actuation matrix, $\boldsymbol{\tau} \in \mathbb{R}^{n}$ is the vector of applied joint torques, and $\mathbf{J}_{p_{i}}(\mathbf{q})$ is the contact Jacobian at the point $\mathbf{p}_{i}$, where the contact forces $\mathbf{f}_{i}$ are applied with respect to $\Sigma_{W}$.

In our approach, we calculate the CoP using whole-body centre of mass (CoM) position and acceleration that are obtained from the human model. In the static condition, 

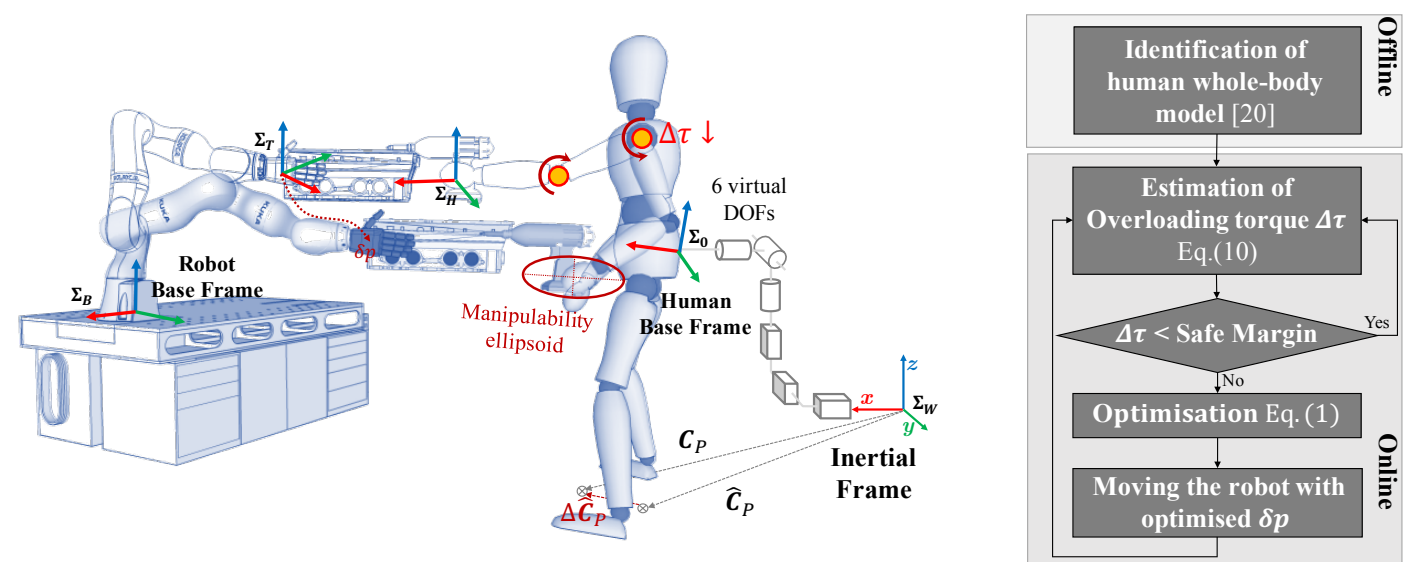

Fig. 2: The floating base whole-body model of the human, the robot model (left) and block diagram of the proposed method (right).

the ground-projected CoM corresponds to the CoP. In the dynamic condition, it is known that the difference between the CoP and the projected whole-body CoM highly correlates with the angular acceleration of the body [20]. Deriving from this, we can obtain the CoP components on the contact surface in the dynamic condition as [21]

$$
C_{P x}=C_{M x}-\frac{\left(C_{M z}-C_{P z}\right)}{\ddot{C}_{P z}+g} \ddot{C}_{M x}
$$

and

$$
C_{P y}=C_{M y}-\frac{\left(C_{M z}-C_{P z}\right)}{\ddot{C}_{P z}+g} \ddot{C}_{M y},
$$

where $g$ is gravitational constant, $C_{P x}$ and $C_{P y}$ are positions of the CoP in x and y axis, $C_{P z}$ is the height of ground and $\mathbf{C}_{M}=\left[\begin{array}{lll}C_{M x} & C_{M y} & C_{M z}\end{array}\right]^{T} \in \mathbb{R}^{3}$ is CoM vector. If the ground is flat and not moving with respect to $\Sigma_{W}$, then $C_{P z}$ and $\ddot{C}_{P z}$ become zero. Therefore, we only consider the second derivative of the CoM vector and $g$ in order to determine the $\mathrm{CoP}$ of the human body. In order to get the acceleration of the CoM vector, we use the Kalman filtering approach [22].

The whole-body $\mathrm{CoM} \mathbf{C}_{M}$ of any branched chain (e.g., leg, arm, etc.) can be defined by geometric parameters (i.e. CoM, mass and length of each link) of the original wholebody structure using the SESC technique as [23]

$$
\mathbf{C}_{M}=\mathbf{x}_{0}+\mathbf{B} \boldsymbol{\Phi},
$$

where matrix $\mathbf{B}=\left[\begin{array}{lll}\mathbf{A}_{0} & \cdots & \mathbf{A}_{n}\end{array}\right] \in \mathbb{R}^{3 \times 3(n+1)}$ contains $i$-th link rotation matrices $\mathbf{A}_{i} \in S O(3)$ with respect to $\Sigma_{W}$. Matrix $\boldsymbol{\Phi}=\left[\begin{array}{lll}\boldsymbol{\phi}_{0}^{T} & \cdots & \boldsymbol{\phi}_{n}^{T}\end{array}\right]^{T} \in \mathbb{R}^{3(n+1)}$ includes the vector of SESC parameters $\boldsymbol{\phi}_{i} \in \mathbb{R}^{3}$, which refer to mass distribution of the human model. In order to identify subject-specific geometric parameters $\hat{\boldsymbol{\Phi}}$, we use classical least-square approach same as in [18]. By using $\mathbf{C}_{M}$ from (4) in (3), we can get a realtime estimation of CoP vector $\mathbf{C}_{P_{w o}} \in \mathbb{R}^{2}$.

The overloading joint torque vector can be calculated from the difference between the CoP $\hat{\mathbf{C}}_{P_{w o}}$ estimated solely from the SESC model and the measured $\mathrm{CoP} \mathbf{C}_{P_{w t}}$ that also includes the external load, as in [18]. However, in this case an external sensory devices, such as force plate or sensorised insoles, are required to obtain the measured CoP. To reduce the complexity and increase the applicability of our approach in human-robot co-manipulation tasks, we propose an extension of SESC parameters that describes the external object manipulated by the human. Based on this parameter we can calculate the CoP of human body with the external load and use it instead of the measured CoP. This approach is feasible when the robot can estimate the parameters of an unknown external object by its own sensory system (i.e. before handover), or when the used tool in comanipulation is known in advance due to the predefined production process and therefore its parameters can be preestimated.

Modified SESC parameters $\hat{\boldsymbol{\Phi}}_{M} \in \mathbb{R}^{3(n+1)}$ can be expressed as

$$
\begin{gathered}
\multicolumn{1}{c}{\hat{\boldsymbol{\Phi}}_{M}=\frac{M}{M+m_{e}}\left(\hat{\boldsymbol{\Phi}}+m_{e} \mathbf{D}_{e}\right),} \\
\mathbf{D}_{e}=\left[\begin{array}{lllllll}
\mathbf{d}_{k}^{T} & \mathbf{0}_{1 \times 3 k} & \mathbf{d}_{k+1}^{T} & \cdots & \mathbf{d}_{k+l}^{T} & \mathbf{c}_{e}^{T} & \mathbf{0}_{1 \times r}
\end{array}\right]^{T},
\end{gathered}
$$

where the total mass $M$ is represented by the sum of the whole-body link masses and $m_{e}$ is the external object mass. $\mathbf{D}_{e}$ represents the forward kinematics of the external object, where $\mathbf{d}_{i} \in \mathbb{R}^{3}$ is the $i$-th link length vector with respect to the previously connected local reference frame, $k$ refers to an index of the local reference frame of the branch (i.e. arm) and $l$ is the number of links in this branch. The position vector of CoM for the external object $\mathbf{c}_{e} \in \mathbb{R}^{3}$, which extends the original branch, is represented with respect to $\Sigma_{H} . r$ is the number the links unrelated to the manipulation of the external object (e.g., legs, etc.). In order to estimate the CoP with externally loaded condition $\hat{\mathbf{C}}_{P_{w t}}$ we perform the calculation of (4) and (3) with the extended model $\hat{\boldsymbol{\Phi}}_{M}$ from (5).

\section{Overloading Joint Torques}

To estimate overloading joint torques, we use the difference between the estimated $\mathrm{CoP} \hat{\mathbf{C}}_{P_{w o}}$ from the human body model without the external object and the $\mathrm{CoP} \hat{\mathbf{C}}_{P_{w t}}$ from body model with the external object. The condition without the external object produces a torque vector

$$
\mathbf{S}^{T} \boldsymbol{\tau}_{w o}=\boldsymbol{\tau}_{b}-\sum_{i=1}^{n_{f}} \mathbf{J}_{\hat{C}_{P_{w o}}}(\mathbf{q})^{T} \mathbf{f}_{w o, i}
$$


where $\tau_{b} \in \mathbb{R}^{n+6}$ is the joint torque vector of human body without any external contact (i.e. without the ground contact as well) and $\mathbf{f}_{w o}$ is the vertical GRF (vGRF) in this condition, which can be estimated by the human body mass. $n_{f} \in$ $\{0, \cdots, f \leq 2\}$ is the number of ground contact points at the foot. On the other hand, the condition with the external object produces a torque

$$
\mathbf{S}^{T} \boldsymbol{\tau}_{w t}=\boldsymbol{\tau}_{h}-\sum_{i=1}^{n_{f}} \mathbf{J}_{\hat{C}_{P_{w t}}}(\mathbf{q})^{T} \mathbf{f}_{w t, i}-\sum_{j=1}^{n_{h}} \mathbf{J}_{a_{h j}}(\mathbf{q})^{T} \mathbf{f}_{h, j} .
$$

where $\mathbf{f}_{w t}$ is the vGRF in this condition, which can be estimated by the combined mass of the human body and the external object. $n_{h} \in\{0, \cdots, h \leq 2\}$ is the number of contact points where the interaction forces are applied.

The relationship between the interaction force $\mathbf{f}_{h}$ and vGRF variation $\Delta \mathbf{f}_{w, i}=\mathbf{f}_{w t, i}-\mathbf{f}_{w o, i}$ can be defined as

$$
\Delta \mathbf{F}=\sum_{i=1}^{n_{f}} \Delta \mathbf{f}_{w, i}=-\sum_{j=1}^{n_{h}} \mathbf{f}_{h, j}
$$

We consider an approximate distribution gain for vGRF and interaction forces $\left(0 \leq \zeta_{i}, \eta_{j} \leq 1\right)$. Each gain can be distributed according to the body configuration over the number of contact points $n_{f}$ and $n_{h}$, receptively [24]-[26]. Deriving from (7), (8) and (9), the overloading joint torque can be defined as

$\Delta \boldsymbol{\tau}_{s}=\sum_{j=1}^{n_{h}} \mathbf{J}_{a_{h j}}(\mathbf{q})^{T} \eta_{j} \Delta \mathbf{F}-\sum_{i=1}^{n_{f}}\left(\mathbf{J}_{\Delta C_{P i}}(\mathbf{q})^{T} \mathbf{f}_{w o, i}+\mathbf{J}_{\hat{C}_{P i}}(\mathbf{q})^{T} \zeta_{i} \Delta \mathbf{F}\right)$,

with the Jacobian of the CoP displacement defined as $\mathbf{J}_{\Delta C_{P_{w o} i}}=\mathbf{J}_{\hat{C}_{P_{w t i}}}-\mathbf{J}_{\hat{C}_{P_{w o} i}}$. It is important to note that $\tau_{b}$ does not affect the overloading joint torque vector $\Delta \tau_{s}$ in any configuration.

\section{Robot Control}

By using (10) in the optimisation process (1) the robot can calculate the optimised configuration of the human. This configuration is then used by the robot to control the location of co-manipulation or handover task execution. The robot was controlled by a Cartesian impedance controller [27]. The translational stiffness was set to $1500 \mathrm{~N} / \mathrm{m}$ in all axes. The rotational stiffness was set to $150 \mathrm{Nm} / \mathrm{rad}$ in all axes. These values provide a reasonable trade-off between the robot tracking performance and the achieved end-effector compliance, in case of a collision.

\section{EXPERIMENTS}

In the experiments we focused on co-manipulation and handover tasks. In these tasks the robot should adapt its behaviour in a way that the working conditions are as optimal as possible for the human co-worker to prevent any excessive joint load and maximise the arm manipulation capacity. In the co-manipulation task, the human arm should ideally work around the configurations where joint torques are low, and at the same, where the manipulability is high enough to facilitate an effective manipulation. In the handover task, the robot should choose the handover location in a way to
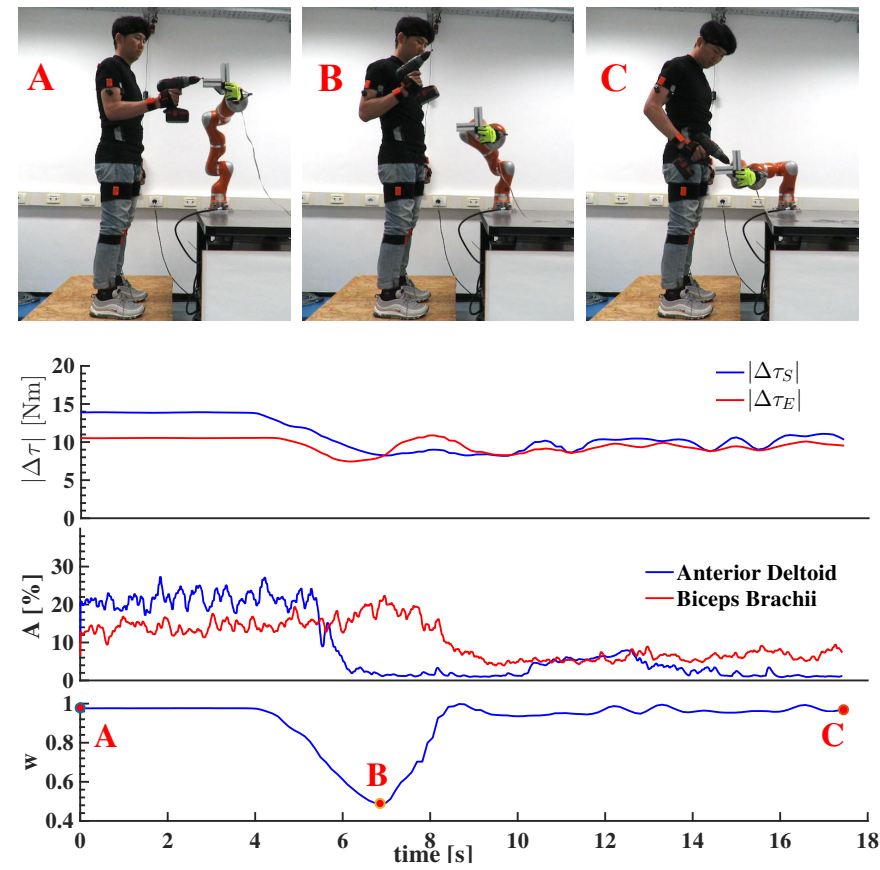

Fig. 3: Results of co-manipulation experiments. The sequence of the photos on the top shows the progress of the experiment: unoptimised state (A), transition (B) and optimised state (C). The first plot shows estimated overloading joint torques in the human arm. The second plot shows muscle activity in the arm as measured by EMG. The third plot shows the human arm manipulability.

minimise the stress on the human joints after the transfer of an object with heavy weight. The manipulability constraint was set to $w_{\min }=0.99$. This ensured that the co-manipulation location was selected within the area where the human arm endpoint had close-to-isotropic manipulability ellipsoid, which facilitated a good force and velocity production capacity in all directions. Please refer to the supplementary multimedia file for a video of experiments.

One subject participated in the experiments (mass $=77 \mathrm{~kg}$ and height $=178 \mathrm{~cm}$ ). For these experiments we used $5 \mathrm{DoF}$ model and focused on sagittal plane. Note that more DoF in 3D space can be used within the existing framework if more complexity is required. Subject-dependent model parameters were estimated before experiments and were then used for the on-line estimation and optimisation during experiments (details of the identification experiment can be seen in [18]). To measure the human kinematics on-line we used a wearable MVN Biomech suit (Xsens Technologies BV) that is composed of seventeen inertial measurement units (IMU). To compare the muscular effort during the task execution between the proposed method and the unoptimised condition, we also measured the muscle activity in the human arm by electromyography (EMG) using Delsys Trigno Wireless system. We measured Anterior Deltoid (AD) and Biceps Brachii (BB), which are related to shoulder and elbow flexion and are stressed the most in the selected tasks. The EMG signals were processed by rectification and low-pass filtering and were normalised to maximal voluntary contraction. 


\section{A. Co-Manipulation Task}

The task of the human in this experiment was to use a hand-held polishing device to polish an object with a complex surface (L-shaped object with a groove, see Fig. 3). The task of the robot was to hold the object and move it to the location where it is the most efficient for the human to perform the polishing. The location was determined by the optimisation process in (1) based on the reduced overloading joint torques and human arm manipulability constraint. The overloading joint torques and the manipulability were calculated based on the tool end-effector by including the tool into the extended model by (5). The mass of the tool was $3 \mathrm{~kg}$ and the length was $20 \mathrm{~cm}$. Since the tools used in the production process are usually known, including the tool into the model beforehand is feasible. We performed the task three times.

The results of experiments are shown in Fig. 3. The left photo (A) in the first row depicts the unoptimised configuration. In the middle photo (B) the human retracted the tool to allow the robot to move to the optimised position. In the right photo $(\mathrm{C})$ the robot moved the object in the optimised configuration and the human performed the polishing task. The subsequent plots show the estimated overloading human joint torques and the measured muscle activity of human arm, respectively. The plot in the last row shows the human arm endpoint manipulability $w$ measure throughout the experiment. The average overloading torques in the unoptimised condition were: $13.9 \pm 0.02 \mathrm{Nm}$ in the shoulder and $10.5 \pm 0.01 \mathrm{Nm}$ in the elbow. In the optimised condition the torques were: $9.7 \pm 0.7 \mathrm{Nm}$ in the shoulder and $9.1 \pm 0.4 \mathrm{Nm}$ in the elbow. The average muscle activities in the unoptimised condition were: $21.5 \pm 2.1 \%$ in $\mathrm{AD}$ and $14.2 \pm 1.6 \%$ in BB. In the optimised condition the activities were: $3.4 \pm 2.0 \%$ in $\mathrm{AD}$ and $6.0 \pm 1.1 \%$ in $\mathrm{BB}$. While the manipulability was in this particular case high in both configurations (A and $\mathrm{C}$ ), the reduction of the shoulder and elbow joint torque were average for $30.3 \%$ and $13.3 \%$ respectively, in the optimised configuration (C) compared to the initial configuration (A). In the meantime, the muscle activity of $\mathrm{AD}$ and $\mathrm{BB}$ reduced for $84.2 \%$ and $57.6 \%$ respectively, thus providing more ergonomic working conditions for the human co-worker. Note that the manipulability temporarily decreased when the human retracted the tool in the transition phase (B).

\section{B. Handover Task}

The task of the human in this experiment was to take an object from the robot and transport it to a predefined place. The task of the robot was to bring the object to the human and hand it over in a location where the predicted overloading human joint torques caused by the object will be minimised according to (1). The mass of the object was $2 \mathrm{~kg}$ and it was estimated on-line by the robot force/torque sensory system. Contrary to co-manipulation task example, where the tool is normally defined and can be pre-measured, the unknown objects in a handover task can vary and therefore it is beneficial if the robot can estimate their properties
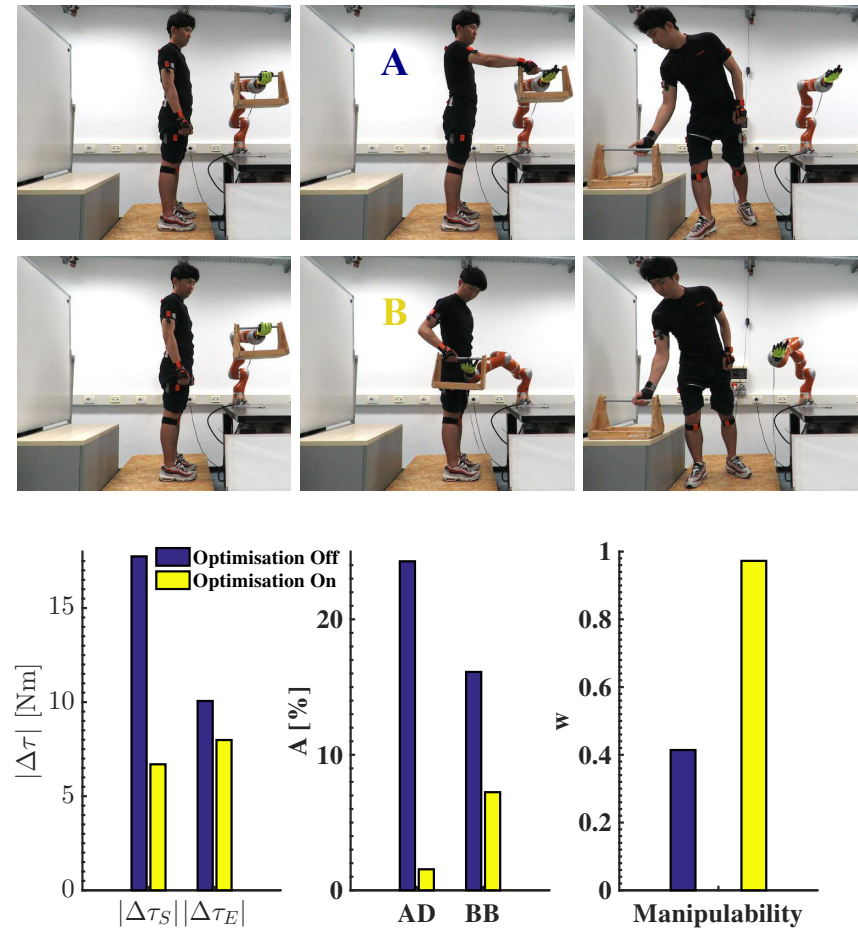

Fig. 4: Results of handover experiments. The sequence of the photos on the top shows the progress of the experiment. The photos in the first row show the handover in the unoptimised condition (A), while the photos in the second row show the handover in the optimised condition (B). The graphs in the bottom row show the average overloading joint torque, muscle activity and arm manipulability of the human, respectively. The blue bars show results for the unoptimised condition, while the yellow bars show results for the optimised condition.

on-line. After the robot measured the object properties, it also included it into the extended model (5). We performed two trials for this task. In the first trial the robot handed over the object in the unoptimised location. In the second trial the robot used the proposed method to estimate the appropriate handover location where the human joint torques were minimised and the arm manipulability was maximised. Each trial was repeated three times.

The results of experiments are sown in Fig. 4. The sequence of photos in the first row shows the handover task execution in the unoptimised location (A), while the sequence of photos in the second row shows the task execution in the optimised location (B). The plots in the third row show the average overloading joint torques, the average muscles activity and average arm manipulability between unoptimised and optimised conditions. We can see that joint torques and arm muscle activity were significantly decreased by the use of the proposed method. The average overloading torques in the unoptimised condition were: $17.8 \pm 0.3 \mathrm{Nm}$ in the shoulder and $10.1 \pm 0.2 \mathrm{Nm}$ in the elbow. In the optimised condition the torques were: $6.7 \pm 2.1 \mathrm{Nm}$ in the shoulder and $8.0 \pm 0.9 \mathrm{Nm}$ in the elbow. The average muscle activities in the unoptimised condition were: $24.3 \pm 2.6 \%$ in $\mathrm{AD}$ and $16.1 \pm 4.3 \%$ in $\mathrm{BB}$. In the optimised condition the activities were: $1.5 \pm 0.3 \%$ in $\mathrm{AD}$ and $7.2 \pm 1.7 \%$ in $\mathrm{BB}$. The overloading joint torques decreased on average by 62.3 $\%$ in the shoulder and $20.6 \%$ in the elbow. The muscle 
activity decreased on average by $93.6 \%$ in $\mathrm{AD}$ and $55.1 \%$ in BB. In addition, the human arm endpoint manipulability was increased compared to the unoptimised location.

\section{DISCUSSION}

The results of experiments show that the proposed method can be successful in providing more ergonomic working conditions for the human co-worker in human-robot comanipulation and handover tasks. The robot was able to find a configuration of the human body where overloading joint torques were decreased and the human arm endpoint manipulability was reasonably high to enable a good manipulation capacity. The muscle activity as measured by EMG confirmed the decrease of overloading joint torque. However, there was some difference between reduction rate of the overloading torques and the muscle activity. This difference can probably be attributed to arm being actuated by a large amount of muscles, while we measured only the muscles that have a dominant role in the selected tasks.

One potential limitation of the proposed approach is that it requires identification of human model parameters for each worker. However, this process has to be done only once and the identified parameters can be reused if there are no considerable changes to the human body. Another potential limitation is that the robot has to measure the human kinematics. In these experiments we used relatively expensive and complex motion capture system. However, the motion capture system can be replaced with more affordable and less complex systems (e.g., Microsoft Kinect).

In future, we will extend the current work to include a more complex model of the human arm manipulability, which takes into account non-linear characteristics of human muscles [28]. In addition, we will perform experiments on multiple subjects executing even more complex motion in 3D space.

\section{REFERENCES}

[1] D. Agravante, A. Cherubini, A. Bussy, P. Gergondet, and A. Kheddar, "Collaborative human-humanoid carrying using vision and haptic sensing," in Robotics and Automation (ICRA), 2014 IEEE Intl. Conf. on, May 2014, pp. 607-612.

[2] K. Kosuge and N. Kazamura, "Control of a robot handling an object in cooperation with a human," in Robot and Human Communication, 6th IEEE Intl. Workshop on, Sep 1997, pp. 142-147.

[3] P. Donner and M. Buss, "Cooperative swinging of complex pendulumlike objects: Experimental evaluation," IEEE Transactions on Robotics, vol. 32, no. 3, pp. 744-753, June 2016.

[4] A. Bicchi and G. Tonietti, "Fast and" soft-arm" tactics [robot arm design]," IEEE Robotics \& Automation Magazine, vol. 11, no. 2, pp. 22-33, 2004.

[5] V. Magnanimo, S. Walther, L. Tecchia, C. Natale, and T. Guhl, "Safeguarding a mobile manipulator using dynamic safety fields," in Intelligent Robots and Systems (IROS), 2016 IEEE/RSJ International Conference on. IEEE, 2016, pp. 2972-2977.

[6] D. Kulić and E. Croft, "Pre-collision safety strategies for human-robot interaction," Autonomous Robots, vol. 22, no. 2, pp. 149-164, 2007.

[7] S. Haddadin, S. Haddadin, A. Khoury, T. Rokahr, S. Parusel, R. Burgkart, A. Bicchi, and A. Albu-Schäffer, "On making robots understand safety: Embedding injury knowledge into control," The International Journal of Robotics Research, vol. 31, no. 13, pp. 15781602, 2012.
[8] A. De Luca, A. Albu-Schäffer, S. Haddadin, and G. Hirzinger, "Collision detection and safe reaction with the DLR-III lightweight manipulator arm," in Intelligent Robots and Systems (IROS), 2006 IEEE/RSJ Intl. Conf. on, Oct 2006, pp. 1623-1630.

[9] J. Kim, A. Alspach, and K. Yamane, "3d printed soft skin for safe human-robot interaction," in Intelligent Robots and Systems (IROS), 2015 IEEE/RSJ Int. Conf. on. IEEE, 2015, pp. 2419-2425.

[10] P. Evrard, E. Gribovskaya, S. Calinon, A. Billard, and A. Kheddar, "Teaching physical collaborative tasks: object-lifting case study with a humanoid," in IEEE-RAS Intl. Conf. on Humanoid Robots, 2009, pp. 399-404.

[11] L. Peternel, T. Petrič, E. Oztop, and J. Babič, "Teaching robots to cooperate with humans in dynamic manipulation tasks based on multimodal human-in-the-loop approach," Autonomous robots, vol. 36, no. 1-2, pp. 123-136, Jan 2014.

[12] H. Ben Amor, G. Neumann, S. Kamthe, O. Kroemer, and J. Peters, "Interaction primitives for human-robot cooperation tasks," in Robotics and Automation (ICRA), 2014 IEEE Intl. Conf. on, 2014, pp. 28312837.

[13] L. Peternel, N. Tsagarakis, D. Caldwell, and A. Ajoudani, "Adaptation of robot physical behaviour to human fatigue in human-robot comanipulation," in IEEE-RAS Intl. Conf. on Humanoid Robots, November 2016, pp. 489-494.

[14] B. M. Nigg, "Biomechanics, load analysis and sports injuries in the lower extremities," Sports Medicine, vol. 2, no. 5, pp. 367-379, 1985.

[15] S. Kumar, "Theories of musculoskeletal injury causation," $E r$ gonomics, vol. 44, no. 1, pp. 17-47, 2001.

[16] S. Gallagher, W. S. Marras, K. G. Davis, and K. Kovacs, "Effects of posture on dynamic back loading during a cable lifting task," Ergonomics, vol. 45, no. 5, pp. 380-398, 2002.

[17] J. C. E. der Burg, J. H. Van Dieen, and H. M. Toussaint, "Lifting an unexpectedly heavy object: the effects on low-back loading and balance loss," Clinical Biomechanics, vol. 15, no. 7, pp. 469-477, 2000.

[18] W. Kim, J. Lee, N. Tsagarakis, and A. Ajoudani, "Human preferences for robot-human hand-over configurations," in 2017 IEEE International Conference on Rehabilitation Robotics (ICORR), Jul 2017, pp. $1-7$.

[19] T. Yoshikawa, "Manipulability of Robotic Mechanisms," The Intl. Journal of Robotics Research, vol. 4, no. 2, pp. 3-9, jun 1985.

[20] D. A. Winter, "Human balance and posture control during standing and walking," pp. 193-214, 1995.

[21] M. B. Popovic, A. Goswami, and H. Herr, "Ground reference points in legged locomotion: Definitions, biological trajectories and control implications," The International Journal of Robotics Research, vol. 24, no. 12, pp. 1013-1032, 2005.

[22] P. R. Bélanger, P. Dobrovolny, A. Helmy, and X. Zhang, "Estimation of angular velocity and acceleration from shaft-encoder measurements," The International Journal of Robotics Research, vol. 17, no. 11, pp. 1225-1233, 1998.

[23] A. González, M. Hayashibe, V. Bonnet, and P. Fraisse, "Whole body center of mass estimation with portable sensors: Using the statically equivalent serial chain and a kinect," Sensors, vol. 14, no. 9, pp. 16955-16971, 2014.

[24] J. J. Yang and J. H. Kim, "Static Joint Torque Determination of a Human Model for Standing and Seating Tasks Considering Balance," Journal of Mechanisms and Robotics, vol. 2, no. 3, p. 031005, 2010.

[25] S. H. Hyon, "Compliant terrain adaptation for biped humanoids without measuring ground surface and contact forces," IEEE Transactions on Robotics, vol. 25, no. 1, pp. 171-178, Beb 2009.

[26] H. Jeong, K. Yamada, M. Kido, S. Okada, T. Nomura, and Y. Ohno, "Analysis of Difference in Center-of-Pressure Positions between Experts and Novices during Asymmetric Lifting," IEEE Journal of Translational Engineering in Health and Medicine, vol. 4, pp. 1-11, 2016.

[27] A. Albu-Schäffer, C. Ott, and G. Hirzinger, "A unified passivitybased control framework for position, torque and impedance control of flexible joint robots," Int. J. Rob. Res., vol. 26, no. 1, pp. 23-39, Jan. 2007.

[28] R. Goljat, J. Babič, T. Petrič, L. Peternel, and J. Morimoto, "Poweraugmentation control approach for arm exoskeleton based on human muscular manipulability," in Robotics and Automation (ICRA), 2017 IEEE Intl. Conf. on, May 2017, pp. 1-6. 